\title{
On the Nonlinear Creep and Recovery of Open Cell Earplug Foams
}

\author{
B. Calcagno, M.d.C. Lopez Garcia, M. Kuhns, and R.S Lakes* \\ Department of Engineering Physics/Department of Materials Science, University of \\ Wisconsin-Madison, 541 Engineering Research Building, 1500 Engineering Drive, Madison, \\ WI 53706-1687 USA
}

Received: 19 March 2008, Accepted: 14 April 2008

\begin{abstract}
The compressive creep and recovery of foam earplugs was studied at various applied stresses leading to different final creep strains. Deformation was determined from digital videos of the earplugs via image processing software. Creep could not be modeled by a single exponential in time; creep approximated a power law in time. Nonlinear viscoelasticity was observed: creep compliance depends on stress level. Nevertheless, for strain as high as $24 \%$, recovery follows creep. Recovery does not follow creep at $70 \%$ strain. Recovery proceeds to completion given enough time.
\end{abstract}

\section{INTRODUCTION}

Polymeric cellular solids, by virtue of the polymeric solid phase, exhibit viscoelastic response. If the solid (neat resin) properties are known and deformation is in the linear range, the foam properties can be calculated via the correspondence principle ${ }^{(1)}$. However, cellular polymers can undergo nonlinear deformation. While the nonlinearity of the stress strain curve ${ }^{(2)}$ is reasonably well characterized and well understood, the nonlinear viscoelastic response is not so well developed. During a compression test of open cell foam, bending of the ribs occur for strains up to 5\%. For strain greater than $5 \%$, the ribs begin to buckle. In this region, the effective compressive modulus is lowered since buckling gives rise to a plateau region as the foam deforms progressively under a constant stress. At sufficiently high compression, the ribs come in contact, the foam densifies, and there is an increase in stiffness.

\footnotetext{
*Corresponding author. bcalcagno@wisc.edu, lakes@engr.wisc.edu, mmkuhns@wisc.edu,mlopezgarcia@wisc.edu

๑Smithers Rapra Technology, 2008
} 
There may also be frictional contact between ribs. In general, below 5\% strain foam behaves in a linear viscoelastic manner. For larger deformations, foam begins to exhibit nonlinearity. For some applications such as earplugs or knee pads, viscoelasticity is essential to the function of the foam.

In a foam earplug, creep behavior is beneficial. The earplug consists of an energy absorbing open cell foam material that is very soft statically, but stiff dynamically, especially in the frequency range of sound waves between $15 \mathrm{~Hz}$ and $15 \mathrm{kHz}^{(3)}$. It is designed to be easily fitted into the ear by controlled viscoelasticity of the polymer. It attenuates sound entering the ear, protecting the ear from damage. According to the US patent ${ }^{(4)}$ of the Classic AEREO safety earplug foams, the compressed plugs recover from $60 \%$ to $40 \%$ compression in about $2-20$ s giving the user enough time to insert it into the ear. The earplugs subsequently attempt to recover their original shape thereby conforming to the structure of the ear canal. Foam earplugs are most commonly made of foamed PVC (poly vinyl chloride). Viscoelastic behavior is governed by an organic plasticizer (aromatic polyester plasticizer and epoxidized soya bean oil stabilizer/plasticizer). In the open cell foam of the earplug, the air phase contributes to stiffness at short time scales. The air does not provide stiffness at long time since it is free to escape through the pores, given enough time. Under a steady load, the air diffuses through the cell walls as the earplug compresses giving rise to creep. Stress induced diffusion of air therefore may play a role in the viscoelastic response. Normal use of the earplug entails nonlinear deformation since the user may roll a $13 \mathrm{~mm}$ diameter plug down to $5 \mathrm{~mm}$ or less in diameter before inserting it into the ear canal. The foam then expands to fill the ear canal, thereby damping noise.

Viscoelastic solids exhibit time dependent behavior which is seen in the strain response to step stress as 'creep'. For viscoelastic solid samples, the strain increases to an asymptotic value. If the stress is removed, the viscoelastic solid follows a delayed recovery path.

The input stress for a creep - recovery protocol is a step up followed by a step down, with $H(t)$ as the Heaviside step function:

$$
\sigma(t)=\sigma_{0}\left[H(t)-H\left(t-t_{1}\right)\right]
$$

For a linear material, the strain is, from the Boltzmann Superposition Principle, with $J(t)$ as the creep compliance:

$$
\varepsilon(t)=\sigma_{0}\left[J(t)-J\left(t-t_{1}\right)\right]
$$

The strain response for time greater than $t_{1}$ is known as recovery. 
The creep compliance $J(t, \sigma)$, allowing for stress dependence which entails a form of nonlinearity, is defined as the ratio of strain to stress:

$$
J(t, \sigma)=\varepsilon(t, \sigma) / \sigma_{0}
$$

If the material is linear, the creep compliance is independent of stress so $J(t$, $\sigma)=J(t)$.

To analyze recovery, the creep curve is fitted with a function, commonly logarithmic or power law, to predict recovery based on Eq. (2). Recovery which obeys Eq. (2), corresponding to a superposition of a delayed and inverted creep curve, is said to "follow creep". Recovery follows creep in linearly viscoelastic materials; also in materials in which the nonlinearity is separable from the time dependence: $J(t, \sigma)=J(t) f(\sigma)$. Such a separable form is referred to as quasi-linear viscoelasticity (QLV).

Nonlinear viscoelasticity theory has been well developed, particularly for application to solid polymers ${ }^{(5,6)}$. Foam materials exhibit nonlinearity in volumetric compression used to determine the bulk modulus ${ }^{(7)}$. Nonlinear viscoelasticity can be described by multiple integral formulations which are highly demanding in terms of experimental input to determine material property functions. In particular, multiple step deformation histories are required to determine the kernel functions in the integrals. Some simplifications to single integrals are known ${ }^{(8,9)}$. For recovery to zero stress, strain recovery will follow creep in a linear material but may not follow creep in a nonlinear material.

In the present study, nonlinear viscoelasticity of earplug foam is studied in the context of stress-dependence of the creep compliance, and the relationship between creep and recovery.

\section{MATERIALS AND METHODS}

As for materials, foam earplugs ("Classic") from AO Safety referred to above were used for all experiments. Figure 1 shows a magnified image of the foam. The average diameter was $13.4 \mathrm{~mm}$, the average height $19.2 \mathrm{~mm}$, and the (calculated) density was $0.108 \mathrm{~g} / \mathrm{cm}^{3}$.

Foam earplugs were tested at ambient temperature $72^{\circ} \mathrm{F}$ in compression via dead weight load that was suspended below the sample with a wire passing through the earplug. This prevented buckling, but required that a 3/32 inch $(2.4 \mathrm{~mm})$ hole be drilled through the center of the earplug. During drilling the earplugs were laterally constrained to ensure the hole was vertical. The sample 


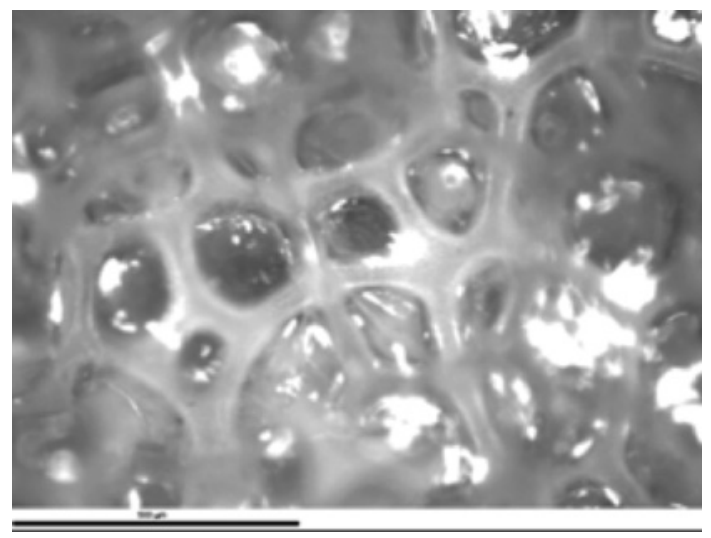

Figure 1. Image of the earplug foam. Scale bar: $0.5 \mathrm{~mm}$

dimensions were measured after allowing the earplug to recover from the stress applied during drilling. A steel music wire of $0.02 "(0.5 \mathrm{~mm})$ diameter certified by standard ASTM-A-228/A, was threaded through the hole so that an attached aluminum plate rested on the foam earplug. Loads of 20, 25, 30, $50,60,80,132$, and 313 grams were hung from the wire causing compression of the earplug by pulling down on the plate. The samples were placed onto a metal stand with space underneath to place and remove the weights. At the loads used, deflection in the steel wire and aluminum plate are negligible, therefore all seen deflections are only due to creep in the earplug. Figure 2 depicts an image of the experimental setup.

To study the recovery in the linear and nonlinear range, the weights were rapidly removed at strains of 3\%, 5\%, 9\%, 24\%, 33\%, 70\%, and 78\%. In order to reach the higher strains and keep the displacements in the camera field of view, the samples were cut using a fine scalpel. The shorter samples require less displacement to obtain high strains. The displacements were captured digitally by obtaining a screen capture video with Rendersoft "Cam Studio" v2.0 software of the image obtained by a Pixelink camera model \# PL-A662. During the loading and unloading steps in most experiments the video was set to capture 2 frames/second, otherwise 1 frame every 10 or 300 seconds was used. For some experiments to delineate the short time region, a rate of 10 frames per second was used. An image magnification of $2.75 \mathrm{x}$ from two lenses in series was used; a LUPlan 5 x .0.15 Nikon lens combined with a $0.55 \mathrm{x}$ DS TV Nikon Lens. For the larger strains, $30 \%$ and $70 \%$, an additional concave lens of focal length $-60 \mathrm{~mm}$ was placed between the foam and the objective to increase field of view from $1.6 \mathrm{~mm}$ to $2.2 \mathrm{~mm}$. Videos were created by using 


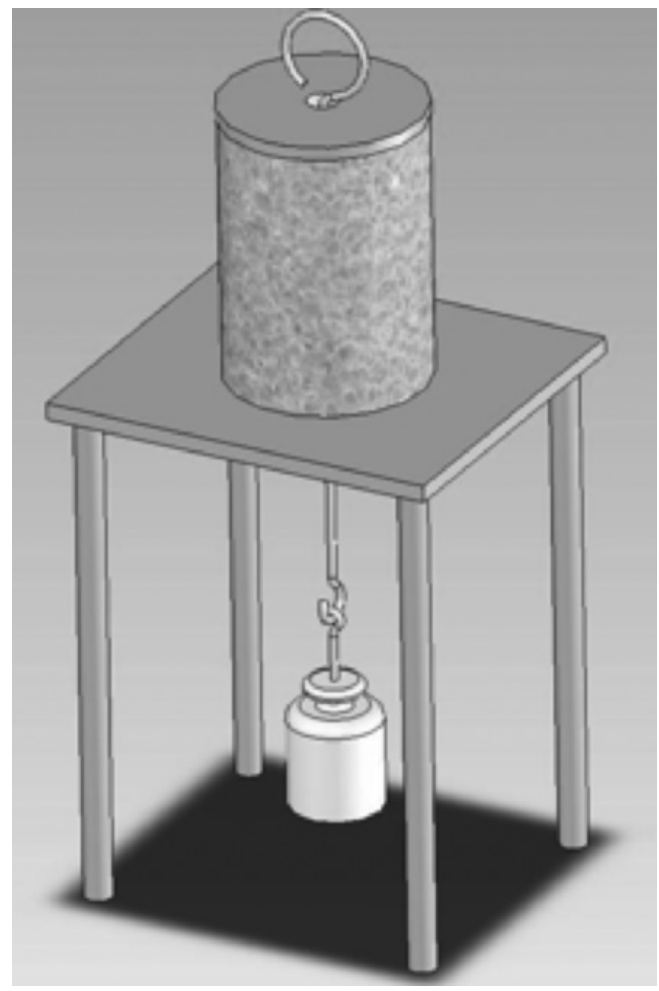

Figure 2. Experimental setup. The dead weight is placed below the earplug to provide stability with respect to buckling. The foam earplug is placed on a platform with a hole to accommodate a wire supporting the dead weight

Cam Studio software to make an image capture of the PixeLink preview screen. The image obtained by Cam Studio was calibrated taking a short video of a calibration slide and measuring the number of pixels corresponding to $0.1 \mathrm{~mm}$ distance using ImageJ software v1.37. The videos were divided into multiple sections using VirtualDub v1.7.6 software and then individual frames were extracted using IrfranView v3.95. The displacements were measured using Image J software.

A displacement value for zero strain was measured from the unloaded earplug in reference to the camera view. This value was subtracted to determine the actual displacement in the earplug for all other measurements. The experimental compliance was calculated using Eq. (3), and a logarithmic or power curve fitting was done to determine the predicted creep and recovery strain via Eq. (2). 


\section{RESULTS AND DISCUSSION}

Many tests were performed at small stresses giving rise to about 5\% strain in anticipation of nonlinear recovery at that threshold. For stresses of $1.5 \mathrm{kPa}$ to $2 \mathrm{kPa}$, creep approximated a power law in time, $\mathrm{t}^{\mathrm{n}}$, with $\mathrm{n}$ from 0.16 to 0.25 . The effective compressive modulus at 10 seconds was about $100 \mathrm{kPa}$. Different earplugs exhibited similar, but not identical, creep. Creep strain curves for different stresses are shown in Figure 3a. As strain approaches the densification region, creep tends to level off. Such behaviour is in contrast to that seen in polycrystalline materials such as metals at high temperature, in which increased stress gives rise to accelerating tertiary creep that can end in rupture. The corresponding creep compliance curves are shown in Figure 3b. Compliance increases with the applied stress, and also becomes more dependent on stress at the larger stresses and at longer times. The shape of each creep curve, not just the magnitude, depends on stress. Therefore the foam material is nonlinearly viscoelastic and does not obey a quasi-linear viscoelastic model (QLV). There are different responses for two pieces cut from the same sample and subject to a stress of $22 \mathrm{kPa}$. This shows the heterogeneity of the earplug foam. Figure 3c displays the strain recovery curves; full recovery to zero occurs even in specimens deformed to large strain.

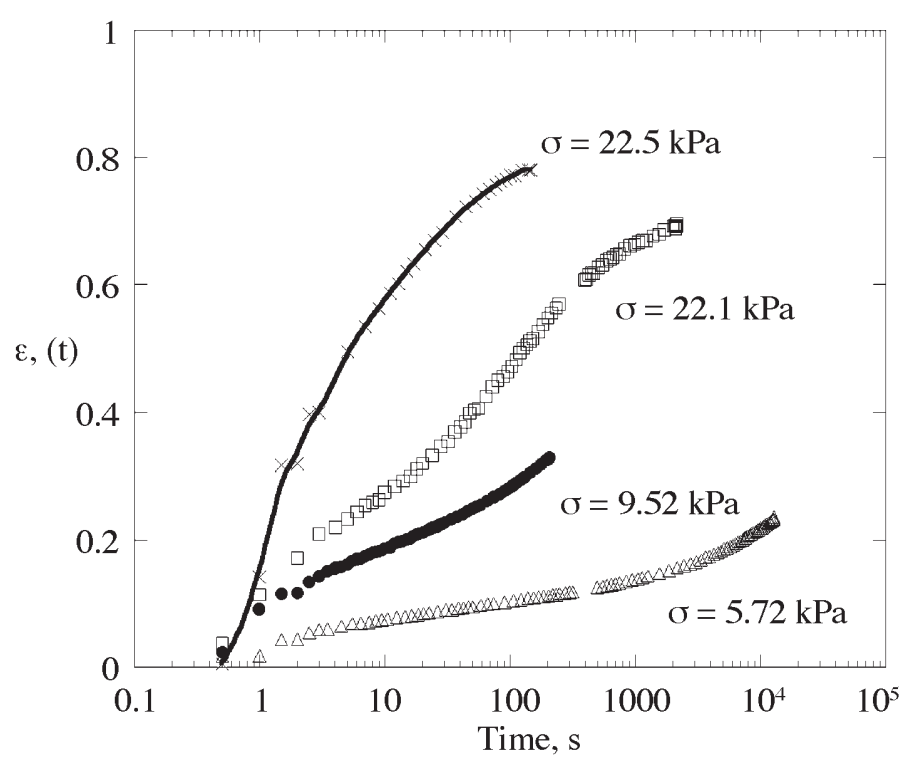

Figure 3a. Creep strain vs. time for various stresses 


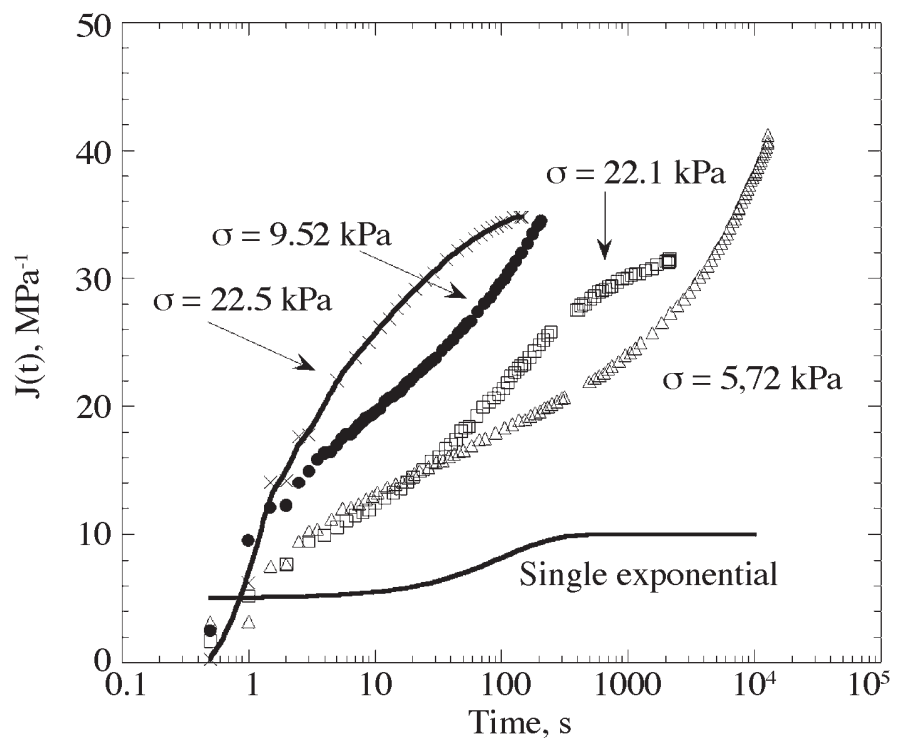

Figure 3b. Creep compliance vs. time for various stresses. Shown for comparison is creep according to a single exponential model

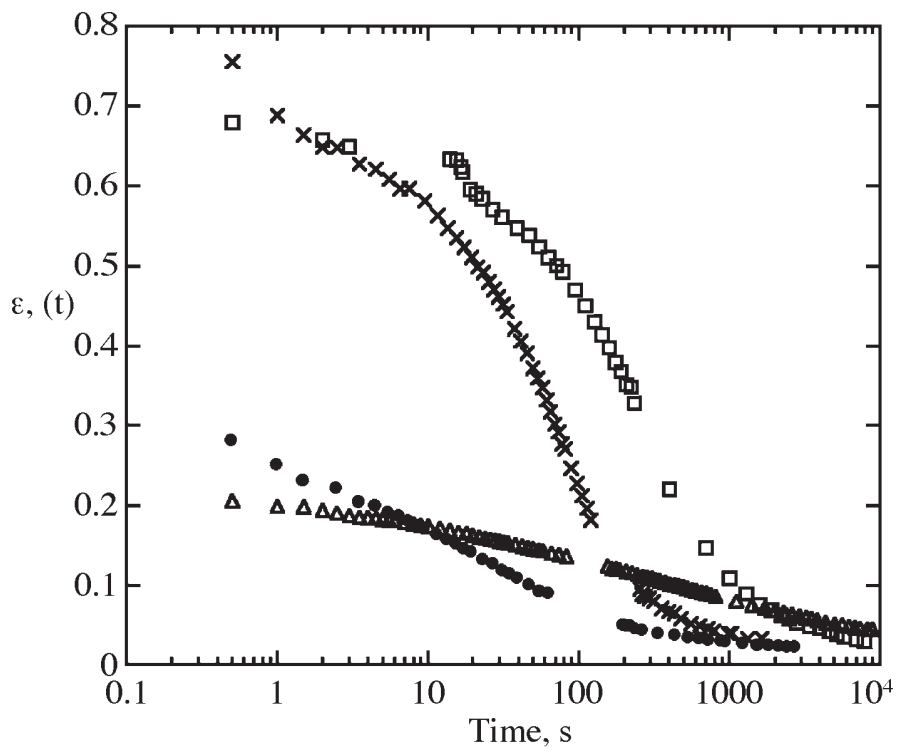

Figure 3c. Summary of results for recovery following creep 
As for comparison of recovery with creep, results of a creep test done with a 4.1 KPa stress reaching approximately 9\% strain are shown in Figures $\mathbf{4 a}$ and $\mathbf{4 b}$. The test lasted 1338 seconds, of which creep took 5.9 seconds and the recovered strain reached $0.01 \%$. The extrapolated creep was calculated from the experimental compliance using a logarithmic curve fit. Figure 4 a shows the extrapolated creep and the experimental data. The predicted and the measured recovery for the test depicted in Figure $\mathbf{4 b}$ show that the recovery follows creep.

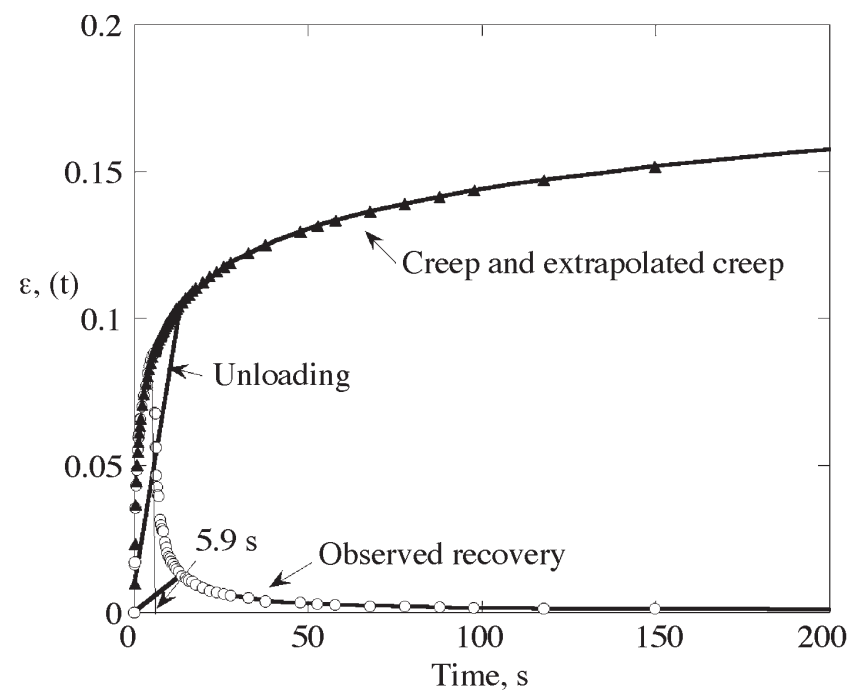

Figure 4a. Experimental creep at a stress $4.1 \mathrm{kPa}$ and recovery (5.9s), and extrapolated creep for a sample taken to $9 \%$ strain

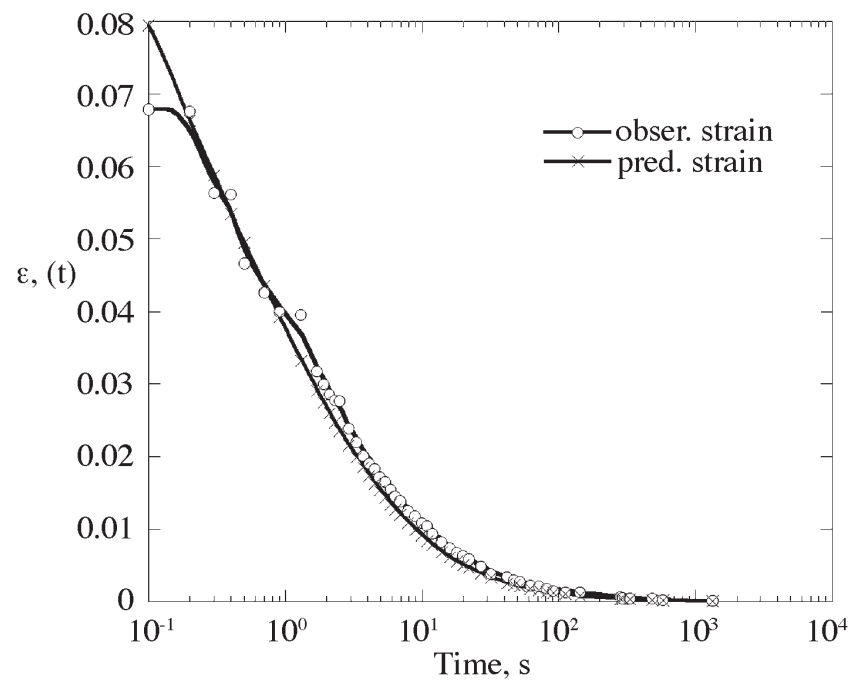

Figure 4b. Experimental and predicted ( $v i a$ a logarithmic fit) strain recovery for sample taken to $9 \%$ strain at a stress $4.1 \mathrm{kPa}$. Recovery follows creep 
Figures 5a and $\mathbf{b}$ show the creep and recovery response during a test in which a sample was subject to $5.7 \mathrm{kPa}$ stress for $12,785 \mathrm{~s}$ reaching almost $24 \%$ strain. Figure 5a shows the experimental creep and recovery, and the extrapolated creep that was obtained using a power fit of the compliance data. For the strain achieved in this test, recovery follows creep as shown in Figure 5b. The sample

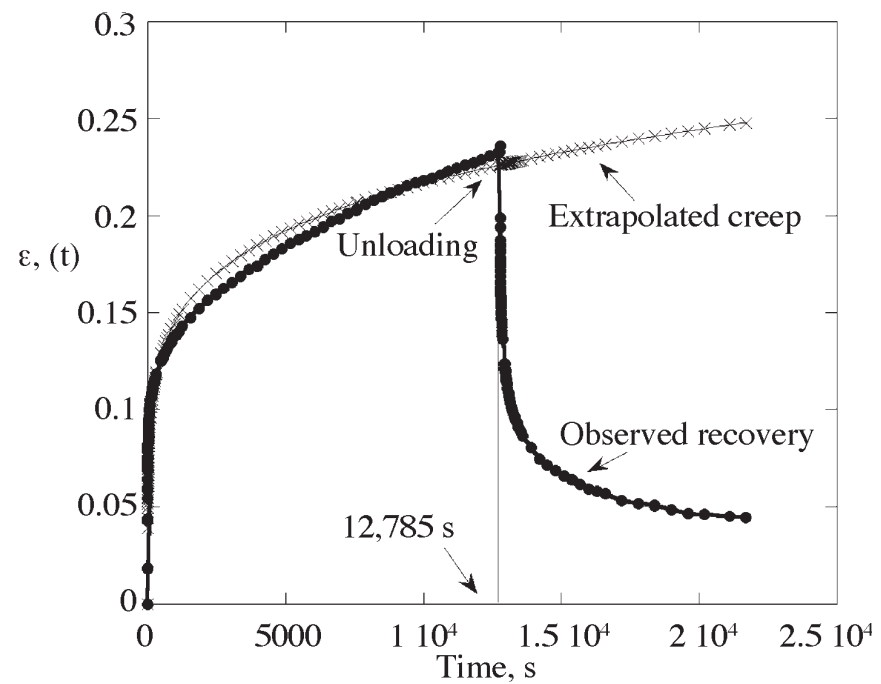

Figure 5a. Experimental creep and recovery, and extrapolated prediction of creep for sample taken to $24 \%$ strain at a stress $5.72 \mathrm{kPa}$. The black line shows time at which load was removed $(12,785 \mathrm{~s})$

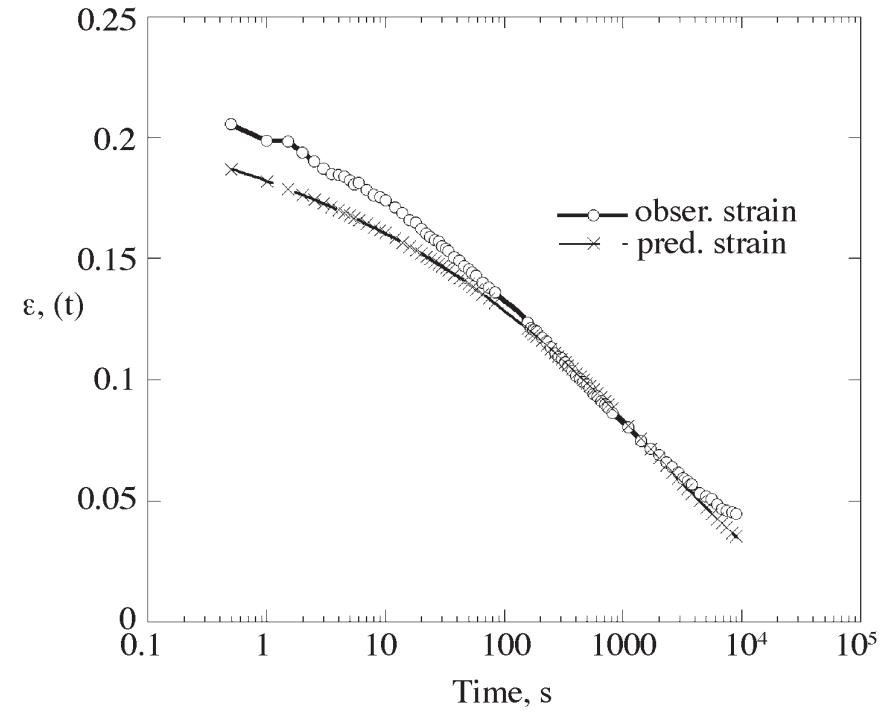

Figure 5b. Experimental and predicted (via a power law fit) strain recovery for sample taken to $24 \%$ strain at a stress $5.72 \mathrm{kPa}$. Recovery follows creep 
used for the previous test was retested at a higher stress after letting it recover for several days. A stress of $9.5 \mathrm{kPa}$ was applied for $208 \mathrm{~s}$, resulting in a final strain of $33 \%$. The creep and recovery strain, as well as the predicted strain calculated using a logarithmic curve fit are shown in Figure 6a. Figure 6b displays the predicted and experimental recovered strain. There is a slight gap between these curves; recovery is slightly slower than creep.

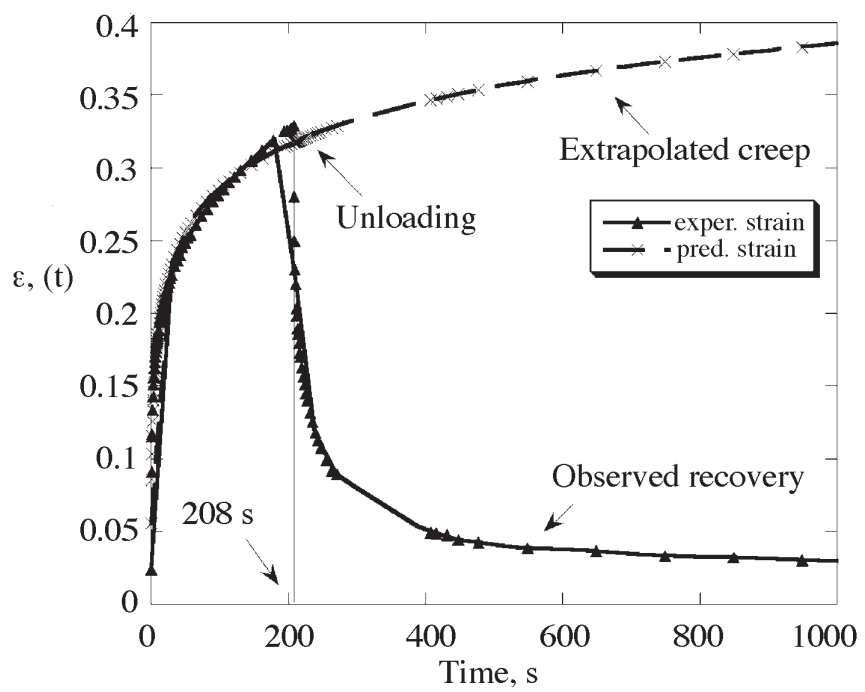

Figure 6a. Experimental creep and recovery, and extrapolated prediction of creep for sample taken to $33 \%$ strain at a stress $9.52 \mathrm{kPa}$. The black line shows time at which load was removed (208s)

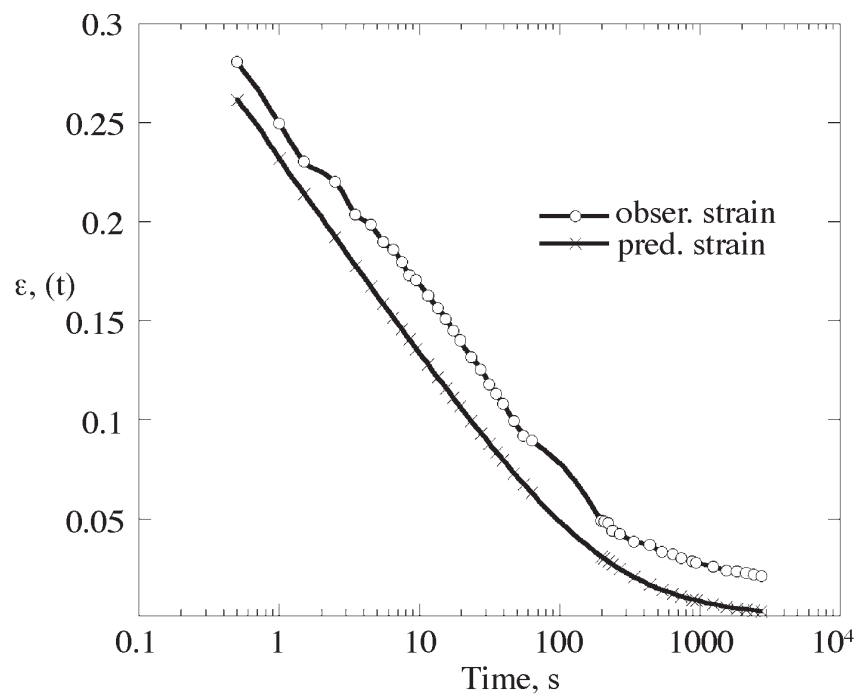

Figure 6b. Experimental and predicted ( via a logarithmic fit of creep) strain recovery for a sample taken to $33 \%$ strain at a stress $9.52 \mathrm{kPa}$. Recovery follows creep 
The sample was cut to produce shorter cylinders so that displacement associated with large strain could be accommodated by the camera. The role of Poisson's ratio in deformation of such samples was considered unimportant since the Poisson's ratio tended to zero at large strain in compression. Both pieces were tested with a load of $313 \mathrm{~g}$. The actual stresses for both samples were $22.5 \mathrm{kPa}$ and $22.1 \mathrm{kPa}$ due to a slight difference in the foam diameter. Figure 7a shows the experimental creep and recovery for the $22.5 \mathrm{kPa}$ stress, which was applied for $147 \mathrm{~s}$. For the predicted creep strain a logarithmic fit was used. The predicted and experimental recovery plotted in Figure 7b show a non-linear behavior in which the recovery does not follow creep. Similar results were obtained in the test of the second sample.

As for the role of stress-induced air-flow in the open cell foam, creep due to such a mechanism ${ }^{(10)}$ predominantly follows a single exponential over a range of time; such effects are known in foams ${ }^{(11)}$. Most of the creep due to a single exponential is complete within one logarithmic decade (a factor of ten) of the time scale as shown in Figure 3b as $J(t)=10\left(\mathrm{MPa}^{-1}\right)\left(1-0.5 \mathrm{e}^{-\mathrm{t} / 100 \mathrm{~s}}\right)$. Since the creep in Figure 3 occurs over at least four decades of time, the primary cause cannot be stress-induced air-flow or any other process which gives rise

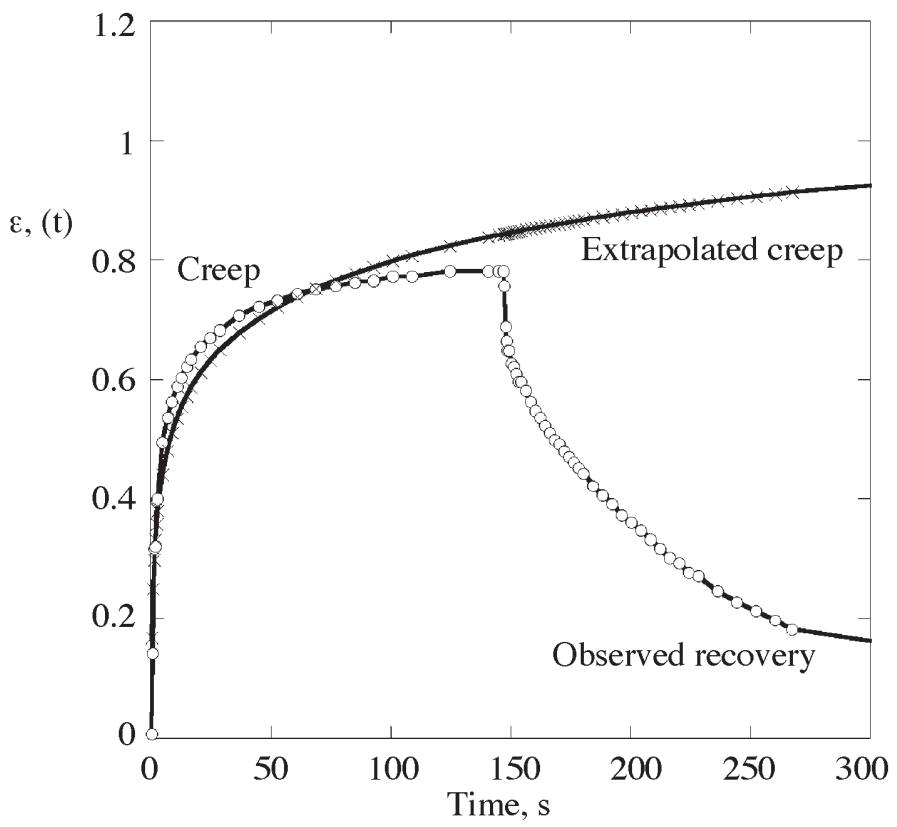

Figure 7a. Experimental creep and recovery, and extrapolated prediction of creep for sample taken to $78 \%$ strain at a stress $22.5 \mathrm{kPa}$. Load was removed at $147 \mathrm{~s}$. A logarithmic function was used to fit creep 


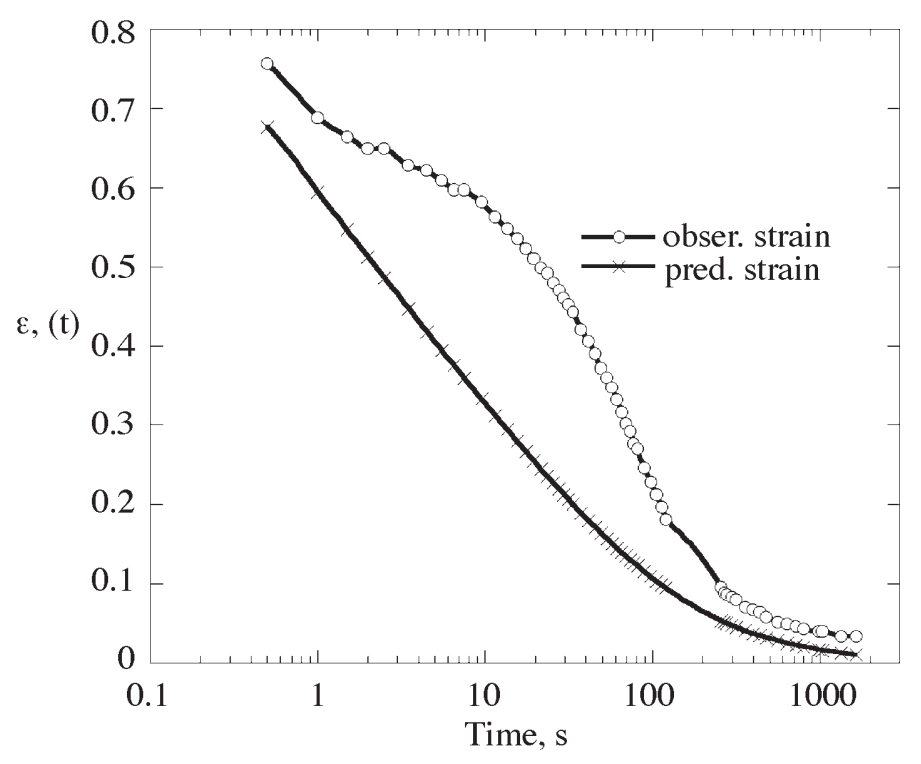

Figure $7 \mathrm{~b}$. Experimental and predicted strain recovery for sample taken to $78 \%$ strain at a stress $22.5 \mathrm{kPa}$. Recovery does not follow creep

to a single exponential. The earplug foam does not have a single recovery time as suggested in the patent. Moreover, air at atmospheric pressure (100 kPa) contributes a short term Young's modulus on the order $100 \mathrm{kPa}$. For the earplug foam, the effective modulus attains such a value after a few seconds of creep. This means stress-induced air flow may play a substantive role in the creep for time less than ten seconds, but that the longer term creep is attributed to molecular mobility in the polymer of which the foam is made.

The creep compliance depends on stress level, so the earplug foam is nonlinearly viscoelastic. Nonlinearity in the strain range 5\% to $50 \%$, in common with the nonlinear stress - strain characteristics of foam, is attributed to buckling of the cell ribs. In this region, recovery follows creep in spite of the nonlinearity. The recovery behaviour is to be understood in the context of the fact that the rib strain in foam is much less than the macroscopic strain, and the fact that the elastomer used in the foam can reversibly undergo substantial strain. Leveling off of the creep at the highest strain is attributed to densification in which cell ribs come into contact. In this region, making contact is not to be expected to be symmetric with breaking contact, hence recovery proceeding more slowly than creep. 


\section{CONCLUSIONS}

Earplug foam is nonlinearly viscoelastic: creep compliance increases with stress for strains greater than $10 \%$. Recovery follows creep for strain as large as $24 \%$ and proceeds to completion. At $70 \%$ strain, recovery does not follow creep, in contrast to the prediction based on linear or quasilinear viscoelasticity. Full recovery occurs after sufficient time even following deformation at $70 \%$ strain.

\section{ACKNOWLEDGEMENTS}

The authors acknowledge the following collaborators: Dr. W. Crone for providing access to the Solid Mechanics Lab; Lei Zhou, for his help setting up the experiments. The authors acknowledge the National Defense Science and Engineering Graduate Fellowship (NDSEG-DOD), for financial support of B. Calcagno, the University of Puerto Rico at Mayaguez from where B. Calcagno is on leave of absence; the Jenkins Predoctoral Fellowship Program (JPFP-NASA) and the Graduate Engineering Research Scholars (GERS), UW-Madison for financial support of M. Lopez Garcia; and the Petroleum Research Fund for support of R. Lakes.

\section{REFERENCES}

1. Lakes R.S., Viscoelastic Solids, CRC-LLC, Boca Raton, 1998.

2. Gibson L.J. and Ashby M.F., Cellular Solids, Structure and Properties, 2nd Edition; Press Syndicate of the University of Cambridge, Cambridge, 1997.

3. Gardner Jr., R. and Berger E.H., History and development of the EAR foam earplug, In: 127th Meeting of the Acoustical Society of America J. Acoust. Soc. Am., 95(5), Pt. 2, p. 2914 Boston, MA 1994.

4. Gardner Jr. R., Earplugs, US Patent 3,811,437 1974.

5. Lockett F.J., Nonlinear Viscoelastic Solids, Academic Press, London, 1972.

6. Ward I. M. and Wolfe J.M., The non-linear mechanical behaviour of polypropylene fibers under complex loading regimes, J. Mech. Phys. Solids, 14, (1966), 131-140.

7. Gardner Jr. R. and Berger E.H., History and development of the EAR foam earplug, In: Meeting of the Acoustical Society of America J. Acoust. Soc. Am., Boston, MA 1994.

8. Pipkin A.C. and Rogers T.G., A non-linear integral representation for viscoelastic behaviour, J. Mech. Phys. Solids, 16, (1968), 59-72. 
9. Schapery R.A., On the characterization of nonlinear viscoelastic materials, Polymer Engineering and Science, 9(4), (1969), 295-310.

10. Biot M.A., General theory of three-dimensional consolidation, J. Applied Physics, 12, (1941), 155-164.

11. Gent A.N. and Rusch K.C., Viscoelastic behavior of open cell foams, Rubber Chemistry and Technology, 39, (1966), 388-396. 